Volume 9, No.1.1, 2020

International Journal of Advanced Trends in Computer Science and Engineering

Available Online at http://www.warse.org/IJATCSE/static/pdf/file/ijatcse2391.12020.pdf

https://doi.org/10.30534/ijatcse/2020/2391.12020

\title{
Electroencephalogram Features Based on One Dimensional Local Binary Pattern
}

\author{
Safie S I ${ }^{1}$, Aimullah Taib ${ }^{2}$, Rosuhana Rahim ${ }^{3}$ \\ ${ }^{1}$ Universiti Kuala Lumpur, Malaysian Institute of Industrial Technology, Johor, Malaysia \\ ${ }^{2}$ Universiti Kuala Lumpur, Malaysian Institute of Industrial Technology, Johor, Malaysia \\ ${ }^{3}$ Universiti Kuala Lumpur, Malaysian Institute of Industrial Technology, Johor, Malaysia
}

\begin{abstract}
This paper discusses the extraction process, to generate unique features of an Electroencephalogram (EEG) signal, using a one-dimensional local binary pattern (1D-LBP) technique. For recognition and classification application, the proposed method has been used to describe the characteristics of an EEG signal. The results of this technique have shown that the unique characteristics of the EEG signal can be obtained well. This paper also presents, an attempt to develop a common feature extraction scheme, which can be used to differentiate ECG features from various EEG signal location.
\end{abstract}

Key words : EEG, 1D-LBP, Feature Extraction.

\section{INTRODUCTION}

Electroencephalography (EEG) is a signal that represents brain activity. It can be recorded by placing the electrodes on the subject's scalp. Synaptic activity results from millions of neurons or nerve cells in the brain, producing these electrical signals in the micro-volt ranges. The number of electrodes used in generating the signals varies between 14 and 256 locations on the scalp or face. As a result, various characteristics and results or classifying data can be obtained using different EEG configurations. The use of algorithms for computing and processing purposes, has now become more intensive for processing the recorded EEG from multiple locations simultaneously [1]. Additionally, these signals embed information in different frequency bands known as wave patterns. By analyzing these frequency components, researchers can uncover important information about the activities being processed by the brain [2].

Feature extraction is the first process done by researchers, after a clean EEG recording is obtained, before going through the next stage, which is the classification. These techniques are usually done using techniques such as Fast Fourier Transformation (FFT) [3], Short Time Fourier Transforms (STFT) [4] and Wavelet Transformation (WT) [5,6]. By using specific extraction techniques, it can guarantee high classification results, improve the classifier result and reduce the size of the data without losing its main components. The use of an effective extraction method can prevent the process of developing complex classification structures.

This paper presents a study on basic steps to extract features of the EEG signals. One dimensional local binary pattern (1D-LBP) will be used to extract discriminative features from these EEG signals. The fundamental description of LBP is explained in section 2. Section 3 explained the process in acquiring the EEG features. This includes the explanation of the results and discussion. Section 4 concludes the paper.

\section{LOCAL BINARY PATTERN}

Local Binary Pattern (LBP) has been extensively being used in two dimensional (2-D) image processing due to its simplicity and its capability to describe combination of texture patterns [7-10]. In one dimensional (1-D) LBP, the algorithm is modified from the (2-D) LBP [9-11]. Both techniques share similar steps. First, the neighborhood of data is examined from a signal $x[n]$. Next, each center data within the samples is threshold against the neighboring data. Finally, 1D-LBP code is assigned to the center data. Mathematically, the 1D-LBP of $\mathrm{x}[\mathrm{n}]$ can be mathematically described as:

$$
\begin{aligned}
& \qquad B P_{p}(x[n])= \\
& \qquad \begin{array}{l}
\sum_{r=0}^{\frac{P}{2}-1}\left\{S\left[x\left[n+r-\frac{P}{2}\right]-x[n]\right] 2^{r}\right. \\
\left.+S[x[n+r+1]-x[n]] 2^{r+\frac{P}{2}}\right\}
\end{array}
\end{aligned}
$$

where $S$ [.] is defined as the Sign function, and mathematically written as: 
Safie S I et al., International Journal of Advanced Trends in Computer Science and Engineering, 9(1.1), 2020, 125 - 128

$$
S[x]=\left\{\begin{array}{lll}
1 & \text { for } & x \geq 0 \\
0 & \text { for } & x<0
\end{array}\right.
$$

$\mathrm{P}$ neighboring samples are threshold around the center sample from the neighborhood of $\mathrm{P}+1$ data samples from the signal $\mathrm{x}[\mathrm{n}]$ on length $\mathrm{N}$ for . The Sign function $\mathrm{s}$ [.] transforms the differences to a -bit binary code. The binomial weight applied to each thresholding operation converts the binary code into a unique LBP code.

The LBP code distinguishes the sample of data and its neighbors. Theoretically, for continuous signals, amplitude of the sample data and its neighbors has no different, making the LBP code produce a value of zero. When there is a significant amplitude difference, such as at peak's position, the LBP code will produce a larger value. The local patterns formed from $x$ [i] can be described by the distribution of the LBP codes:

$$
H_{b}=\sum_{\frac{P}{2} \leq i \leq N-\frac{P}{2}} \delta\left(L B P_{P}(x[n]), b\right)
$$

where $\delta(\mathrm{i}, \mathrm{j})$ is the Kronecker delta function, while $\mathrm{k}=1,2, \ldots . \mathrm{n}$ (number of histogram bins). The 1-D LBP operator has been developed and it was shown that it extracts LBP codes from the analyzed signal. These codes can be used as features and applied to signal processing problems. The procedure for extracting a LBP code for neighborhood of samples can be summarized and reiterated in Figure 1. The procedure iterates over all signal segments and can be used to describe a signal segment by a sparser LBP occurrence histogram.

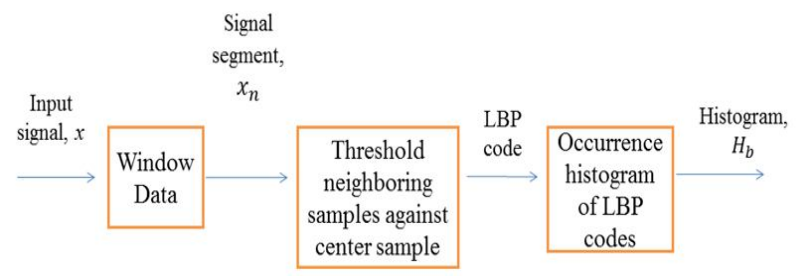

Figure 1: Overview of 1-D LPB procedure on a neighborhood samples to extract a LBP code

\section{SIMULATION SETUP}

The EEG data signals are collected from 30 UniKL student's age between 18 and 30 [12]. All the signals undergo the same pre-processing and feature extraction process before being analysed and compared. The EEG signals will first be filtered to remove un-necessary noises. After removing the unwanted noises in EEG signals, the clean EEG signals needs to be separated into the desired frequency band. The frequency band are divided into five frequency ranges which are from $0 \mathrm{~Hz}$ to $4 \mathrm{~Hz}$, from $4 \mathrm{~Hz}$ to $8 \mathrm{~Hz}$, from $8 \mathrm{~Hz}$ to $16 \mathrm{~Hz}$, from $16 \mathrm{~Hz}$ to $32 \mathrm{~Hz}$ and from $32 \mathrm{~Hz}$ to $64 \mathrm{~Hz}$. They are named as Delta, Theta, Alpha, Beta and Gamma respectively. As for the frequency Bandwidth are 4 for Delta and Theta band, 8 for Alpha band, 16 for Beta band and 32 for Gamma band respectively $[13,14]$. In this studies, Finite Impulse Response (FIR) filter are used to separate the frequency band according studies from [15].

\section{RESULTS}

The result shows the example for five candidates with their respective LBP histogram. As illustrated in Figure 2a, the top figure represents the clean signal of EEG for candidate 1. As for middle figure, shows the segmentation number of LBP feature histogram for the clean signal of EEG candidate one. The segmentation of EEG clean signal followed the studies by [16]. The signal was segmented into 4097 data by using Hanning window (N/4 samples) with no overlaps.

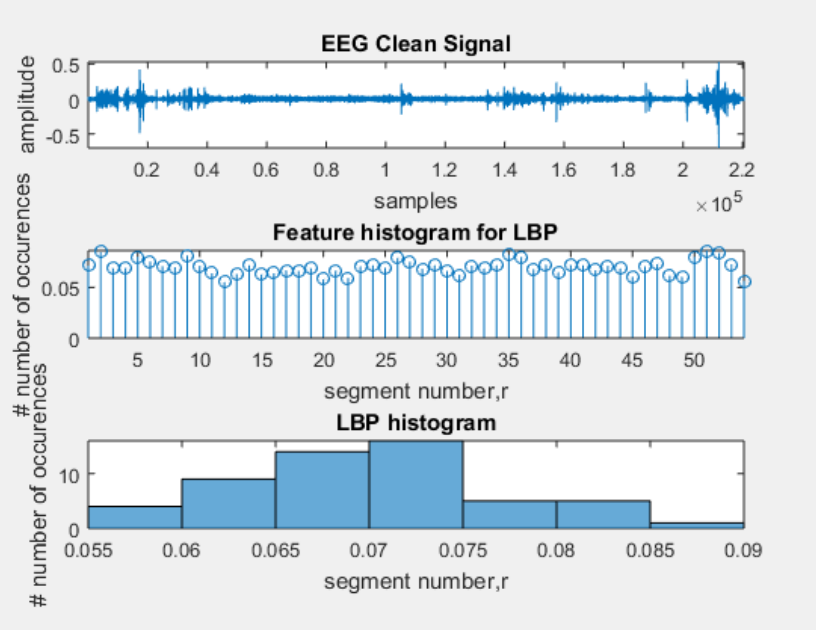

Figure 2a: LBP histogram candidate 1

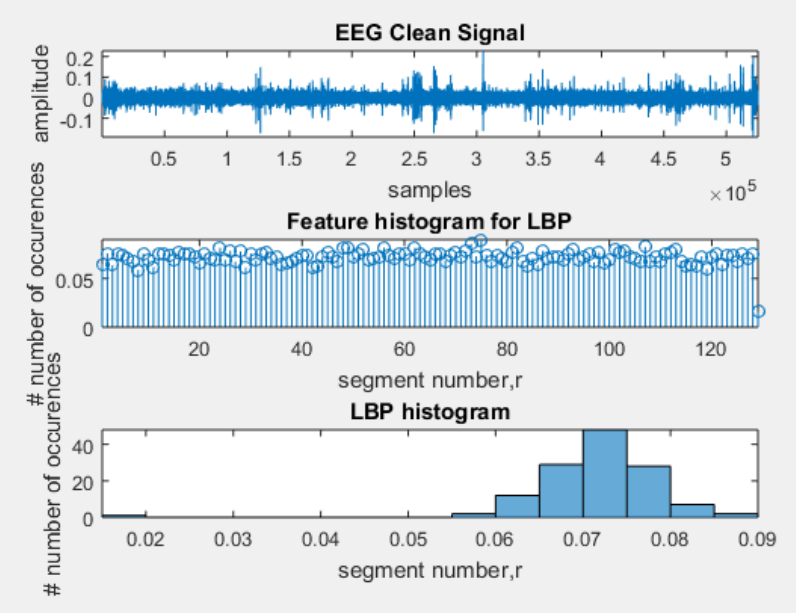

Figure 2b: LBP histogram candidate 2 


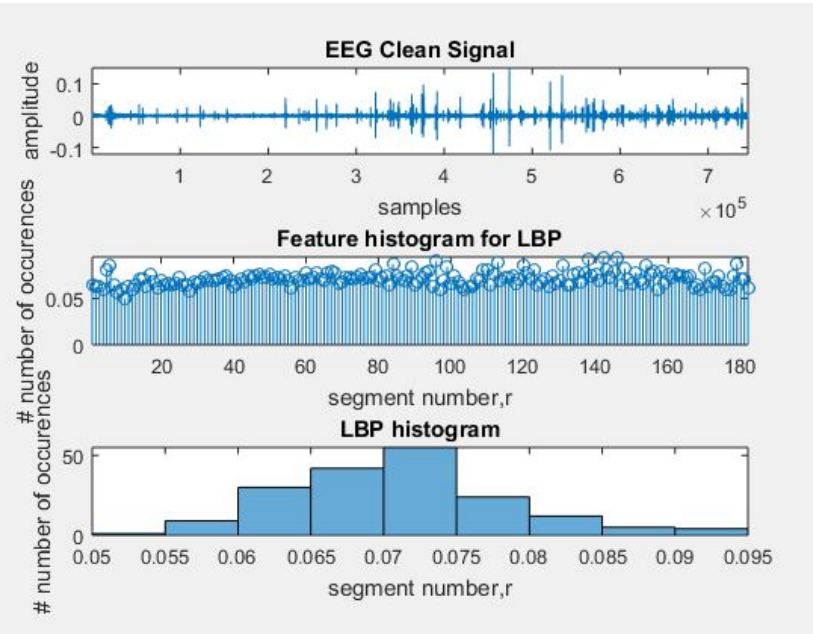

Figure 2c: LBP histogram candidate 3

The value used according to this study will gain the good performances in analyzing EEG signals. After the LBP segment number has been identified, the bottom figure shows the LBP histogram occurrences according to the segmented value.

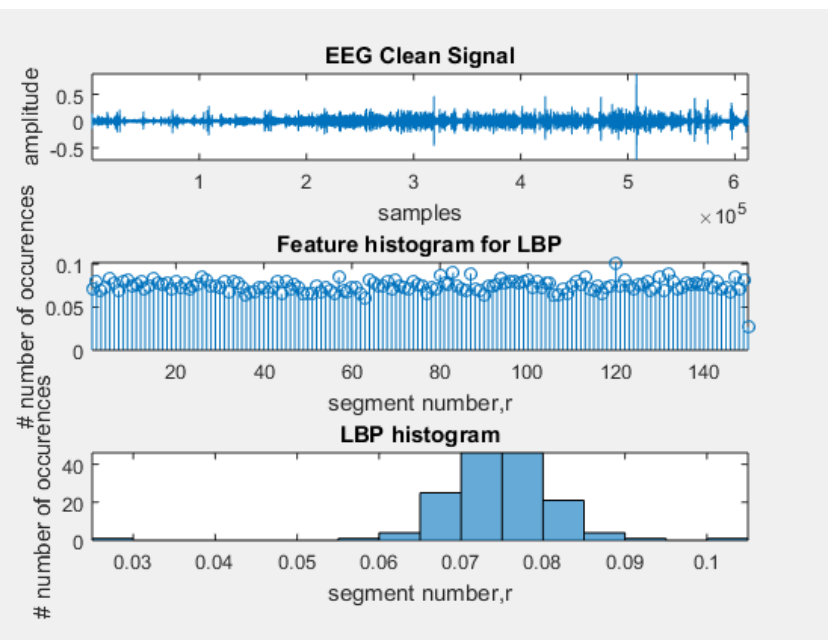

Figure 2d: LBP histogram candidate 4

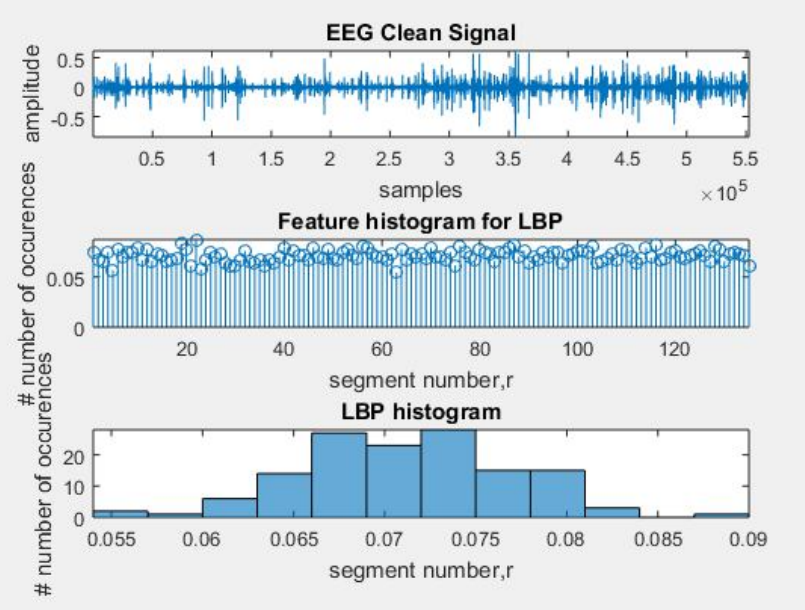

Figure 2e: LBP histogram candidate 5
From this point, the features can be extracted according to the needs of the study. In this study, it only focuses on the value of mean, maximum value and the standard deviation of the LBP histogram. Figures 2b, 2c, 2d, and $2 \mathrm{e}$ follows the same procedure of Figure 2a.

Table 1 shows the obtained EEG features for LBP histogram and the features on the statistical value from one of the signal of EEG. As shown in table 1, the value of standard deviation by candidate $1,2,4$, and 5 have a value that closed to each other. But as for candidate 3 , the value has a slight deviation from other candidates. As for the mean value, all of EEG signals of candidates almost having the same values. As for maximum value, candidate 2 shows the highest value of all candidates followed by candidate 4, 5, 3 and 1 respectively. This shows that simple statistical value of LBP histogram can also be one of the features that can be used to evaluate or analyzing the EEG signal in later stage.

Table 1: obtained EEG features for LBP histogram

\begin{tabular}{llll}
\hline & Std. & Mean & $\begin{array}{l}\text { Maximum } \\
\text { value }\end{array}$ \\
\hline Candidate 1 & 0.0072 & 0.701 & 0.0863 \\
\hline Candidate 2 & 0.0073 & 0.747 & 0.1017 \\
\hline Candidate 3 & 0.0058 & 0.712 & 0.0873 \\
\hline Candidate 4 & 0.0075 & 0.711 & 0.0946 \\
\hline Candidate 5 & 0.0074 & 0.718 & 0.0900 \\
\hline Sum & 0.0070 & 0.718 & 0.0092 \\
\hline
\end{tabular}

\section{CONCLUSION}

This paper presents one of the simplest methods, to extract EEG features, which is by using the 1D-LBP histogram technique. Experimental results indicate that 1D-LBP has the capability to extract unique characteristic from the EEG signal. This technique also demonstrates the ability of the algorithm to be adopted to capture the variations embedded in the signal, which is particularly useful for the process of classifying the EEG signals in the next stage. However, this technique needs to be deeply studied on a larger data set. It is important before this method can be used for clinical purposes. Further planning for this study, is to use the extracted EEG features, for recognizing human emotions.

\section{ACKNOWLEDGEMENT}

The authors would like to thank the Ministry of Higher Education of Malaysia for supporting this research project under the FRGS research grant (FRGS-1-2014-TK03-UNIKL-02-2). 
Safie S I et al., International Journal of Advanced Trends in Computer Science and Engineering, 9(1.1), 2020, 125 - 128

\section{REFERENCES}

1. J. Camacho, V. Manian, Real-time single channel EEG motor imagery based Brain Computer Interface, World Automation Congress (WAC), IEEE, Rio Grande, 2016, pp 1-6.

https://doi.org/10.1109/WAC.2016.7582973

2. J. Chiliński, W. Bauer and J. Baranowski, Bayesian analysis of EEG signal frequency components, $21 s t$ International Conference on Methods and Models in Automation and Robotics, Miedzyzdroje, Poland, 2016, pp. 767-771.

3. H. Mohseni, A. Maghsoudi, M. Kadbi, J. Hashemi, A. Ashourvan, Automatic detection of epileptic seizure using time-frequency distributions, in IET $3 r d$ International Conference on Advances in Medical, Signal and Information Processing, 2006, pp. 1-4. https://doi.org/10.1049/cp:20060378

4. K. Polat, S. Gunes, Classification of epileptiform EEG using a hybrid system based on decision tree classifier and fast Fourier transform, Appl. Math. Comput. 187 (2): 1017-1026, 2007.

5. N. F. Guler, E. D. Ubeyli, Multiclass support vector machines for EEG-signals classification, IEEE Trans. Inf. Technol. Biomed. 11 (2): 117-126, 2007.

6. A. Subasi, EEG signal classification using wavelet feature extraction and a mixture of expert model, Expert Syst. Appl. 32(4): 1084-1093, 2007. https://doi.org/10.1016/j.eswa.2006.02.005

7. B. Kurt, V.V. Nabiyev, Down syndrome recognition using local binary patterns and statistical evaluation of the system, Expert Syst. Appl. 38 (7): 8690-8695, 2011.

8. S. Moore, R. Bowden, Local binary patterns for multi-view facial expression recognition, Comp. Vis. Img. Underst. 115 (4): 541-558, 2011. https://doi.org/10.1016/j.cviu.2010.12.001

9. N. Chatlani, J. J. Soraghan, Local binary patterns for 1-D signal processing, in: 18th European Signal Processing Conference, August 23-27, Aalborg, Denmark, 2010, pp. 95-99.

10. Q. Zhu, N. Chatlani, J. J. Soraghan, 1-D Local binary patterns based VAD used INHMM-based improved speech recognition, in: 20th European Signal Processing Conference, Bucharest, Romania, August 27-31, 2012, pp. 1633-1637.

11. Y. Kaya, M. Uyar, R. Tekin, S. Yıldırım, 1D-local binary pattern based feature extraction for classification of epileptic EEG signals, Applied Mathematics and Computation, 243: 209-219, 2014. https://doi.org/10.1016/j.amc.2014.05.128

12. S. A. Samad, A. B. Huddin, Spectograms and Scalograms with Correlation Filters for Anuran Vocalization Classification. International Journal of Advanced Trends in Computer Science and Engineering, 8 (3): 594-597.
13. K. Q. Lepage, M. A. Kramer, C. J. Chu. A statistically robust EEG re-referencing procedure to mitigate reference effect. Journal of neuroscience methods, 235: 101-116, 2014. https://doi.org/10.1016/j.jneumeth.2014.05.008

14. D. Yao, A comparative study of different references for EEG spectral mapping: the issue of the neutral reference and the use of the infinity reference. Physiological measurement, 26(3): 173-184, 2005. https://doi.org/10.1088/0967-3334/26/3/003

15. B. Sabarigiri, D. Suganyadevi. A Hybrid Pre-Processing Techniques for Artifacts Removal to Improve the Performance of Electroencephalogram (EEG) Features Extraction. International Conference on Innovations in Engineering and Technology, Tamil Nadu, 21st-22nd March, 2014, pp. 2087-2092.

16. B. Boashash, Time-frequency signal analysis and processing: a comprehensive reference. Academic Press, 2015. 